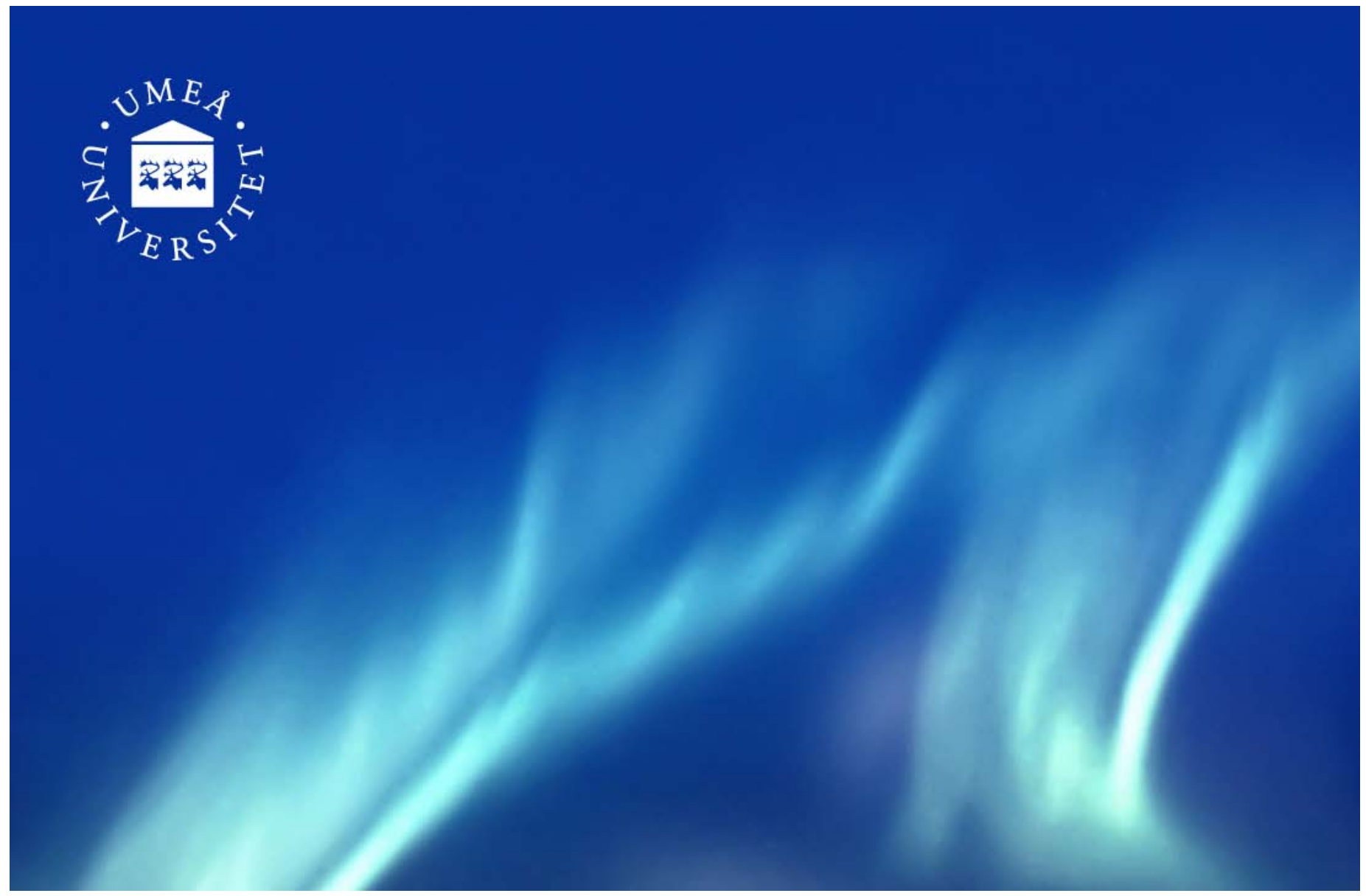

DiVA - Digitala Vetenskapliga Arkivet http://umu.diva-portal.org

This is an author produced version of a paper presented at 2010 Fall Meeting of the Materials Research Society, Boston Nov 29 - Dec 2. This paper has been peer-reviewed but does not include the final publisher proof-corrections or journal pagination.

Citation for the published paper:

Bertil Sundqvist; Ove Andersson; Issam Quwar; Alexandr Talyzin

Low Temperature Phase Diagram of $\mathrm{NH}_{3} \mathrm{BH}_{3}$

Materials research society symposium proceedings: symposium EE, solid-state chemistry of inorganic materials VIII, 2011 Vol. 1309

URL: http://www.mrs.org/f10program-ee/

Access to the published version may require subscription. Published with permission from:

Materials Research Society 


\title{
Low Temperature Phase Diagram of $\mathrm{NH}_{3} \mathrm{BH}_{3}$
}

\author{
Bertil Sundqvist, Ove Andersson, Issam Quwar and Alexandr Talyzin \\ Department of Physics, Umeå University, SE-90187 Umeå, Sweden
}

\begin{abstract}
The pressure-temperature ( $\mathrm{p}-\mathrm{T}$ ) phase diagram of $\mathrm{NH}_{3} \mathrm{BH}_{3}$ has been investigated by thermal conductivity measurements up to $1.5 \mathrm{GPa}$ at temperatures between 100 and $300 \mathrm{~K}$, and the phase boundaries between the three known structural phases have been identified. The transformation between the room temperature tetragonal $14 \mathrm{~mm}$ phase and the low temperature orthorhombic $P m n 2_{1}$ phase $\left(\mathrm{T}_{\mathrm{c}}=218 \mathrm{~K}\right.$ at $\left.\mathrm{p}=0\right)$ shows only a small hysteresis. The transformation into the high pressure orthorhombic $C m c 2_{1}$ phase (at $1.0 \mathrm{GPa}$ near $292 \mathrm{~K}$ ) has a very strong hysteresis, up to $\Delta \mathrm{p}=0.5 \mathrm{GPa}$, and below $230 \mathrm{~K}$ a fraction of this phase is metastable even at atmospheric pressure.
\end{abstract}

\section{INTRODUCTION}

Future hydrogen storage systems for mobile applications should be optimized to contain as much hydrogen as possible, both in terms of volume and in terms of weight [1]. Storage in the form of solid compounds is very efficient, since many such compounds contain more hydrogen per unit volume than pure solid or liquid hydrogen. Therefore, many simple or complex hydrides containing high volume fractions of hydrogen have been studied recently in the search for safe, reversible storage materials. In particular, the temperature-pressure phase diagrams of several complex hydrides have been studied [2-5] to evaluate the possibility to increase the hydrogen density still further by creating structural phases with denser molecular packing.

Ammonium borohydride, $\mathrm{NH}_{3} \mathrm{BH}_{3}$, contains 19.6 percent hydrogen by weight, and the hydrogen can be released in three stages by heating to temperatures in the range $80-500^{\circ} \mathrm{C}[6]$, or by other means. The structural evolution of this material under pressure has recently been studied by several groups [7-9] and three structural phases have been identified in the lowpressure region below 1.5 GPa [8]. Under ambient conditions $\mathrm{NH}_{3} \mathrm{BH}_{3}$ has a tetragonal lattice with symmetry $I 4 \mathrm{~mm}$ and with rotationally disordered molecules aligned with the lattice $\mathrm{c}$ axis. Cooling below about $220 \mathrm{~K}$ results in a transformation into an orthorhombic $P m n 2_{1}$ structure with the molecules tilted by a temperature dependent, small $\left(18-26^{\circ}\right)$ angle relative to the $\mathrm{c}$ axis. Increasing instead the pressure at room temperature causes a transformation near $1.1 \mathrm{GPa}$ into a second orthorhombic structure with $C m c 2_{1}$ symmetry, with strongly inclined molecules $\left(69-79^{\circ}\right.$, increasing with pressure). The high pressure transition is clearly of first order with a $4.4 \%$ volume change, a significant range of phase coexistence (300 MPa) and a large hysteresis in pressure, while the low temperature transition has only a weak first-order character with a volume change of $0.27 \%$ and a $2 \mathrm{~K}$ range of coexistence. Using thermodynamic arguments, 
Filinchuk et al. [8] also predicted the existence of a fourth phase, probably monoclinic $P 2_{1}$, at high pressure and low temperature. Other recent studies have observed further phase transformations at 5 [7], 8 [9] and 12 [7] GPa, and mapped the thermal decomposition behaviour at high temperatures and pressures [10].

In the present work we have used thermal and spectroscopic methods to map the pressure-temperature phase diagram of $\mathrm{NH}_{3} \mathrm{BH}_{3}$ at and below room temperature under pressures up to $1.5 \mathrm{GPa}$, and to search for the predicted $P 2_{1}$ phase. We have not been able to verify the existence of the latter, but we present below an interesting and somewhat complicated phase diagram showing strong hysteresis effects for the high-pressure $C m c 2_{1}$ phase.

\section{EXPERIMENT}

$\mathrm{NH}_{3} \mathrm{BH}_{3}$ with nominal purity $>97 \%$ was obtained from Sigma-Aldrich and used without further purification. The sample source and quality were intentionally chosen to be identical to those used by Filinchuk et al. [8] to enable a direct comparison between the two sets of results. All handling of the material, including filling of the pressure cells, was carried out in a glove-box under dry Ar gas.

To detect phase boundaries we measured the thermal conductivity, $\kappa$, using a dynamic hot-wire method [11] previously used in studies of several other complex hydrides [3,4] as well as many other soft solids [12,13]. In this method, a thin Ni wire is encased in the sample studied, and $\kappa$ is calculated from the measured time dependence of the wire temperature during pulse heating. Measurements on different samples under identical conditions usually differ by $\pm 1 \%$ or less, and the inaccuracy in $\kappa$ is estimated to be smaller than $\pm 2 \%$ under most conditions. Phase transitions are often associated with large changes in $\kappa$ and its temperature dependence, and the heat absorbed/released at transitions also causes characteristic anomalies in the data. Usually, phase boundaries are thus easily found, and quite often it is also possible to identify the phases involved. All thermal measurements were carried out in a steel piston-and-cylinder vessel, 45 $\mathrm{mm}$ in internal diameter, which could be cooled by liquid $\mathrm{N}_{2}$ or heated electrically.

Raman spectra were obtained using a Renishaw 1000 spectrometer with 514 and $633 \mathrm{~nm}$ excitation lasers. High pressure measurements were made in a diamond anvil cell with $0.7 \mathrm{~mm}$ culets. Samples were placed in a $0.3 \mathrm{~mm}$ hole in a stainless steel gasket together with ruby balls for pressure calibration. No pressure transmitting medium was used; this is not expected to modify the measured behavior of complex hydrides because of the low shear strength of the material. Several isobaric cooling experiments down to $240-250 \mathrm{~K}$ were performed, with initial pressures of $0.8,0.9,1.0$ and $1.1 \mathrm{GPa}$.

\section{RESULTS}

To map the pressure-temperature phase diagram we have carried out a large number of (approximately) isothermal pressure runs and isobaric temperature runs, most of these in the range $0.1-1.2 \mathrm{GPa}$ at temperatures between 210 and $300 \mathrm{~K}$. We have also measured $\kappa$ as a function of temperature down to $120 \mathrm{~K}$ at $0.1 \mathrm{GPa}$ and down to $100 \mathrm{~K}$ at $1.5 \mathrm{GPa}$, without finding any additional phase transformations. 
The thermal conductivity was found to show a normal behaviour as a function of temperature, pressure and structure in all the three structural phases observed in the ranges studied. In all phases the thermal conductivity increased with increasing pressure, as should be expected in a crystalline solid. In the rotationally disordered tetragonal $14 \mathrm{~mm}$ phase, near atmospheric pressure and room temperature, $\kappa$ was almost independent of temperature because of strong scattering of phonons by molecular disorder. As shown in figure 1, the transformation into the low temperature $P m n 2_{1}$ structure was associated with a step change in $\kappa$, and in the $P m n 2_{1}$ phase $\kappa$ showed the usual $\kappa \propto \mathrm{T}^{-1}$ dependence associated with phonon-phonon scattering in an ordered, crystalline phase.

In the pressure range up to $0.6 \mathrm{GPa}$ the amplitude of the step change in $\kappa$ at the transition between the tetragonal $14 \mathrm{~mm}$ phase and the low temperature orthorhombic $P m n 2_{1}$ phase increased continuously with increasing pressure (see figure 1). Although there was always a small hysteresis in the transition, corresponding to less than $5 \mathrm{~K}$ or $100 \mathrm{MPa}$ in isobaric and isothermal runs, respectively, the transformation was clearly always reversible and the forward and reverse transitions defined a phase line with a slope of about $60 \mathrm{~K} \mathrm{GPa}^{-1}$ and a zero-pressure transformation temperature of about $218 \mathrm{~K}$, in excellent agreement with the data of Filinchuk et al. [8]. These authors also report that the transformation is close to second order at atmospheric pressure, with a very small volume change. The steady increase in the magnitude of the step in $\kappa$ with increasing pressure observed here indicates that the first-order character of this transition also increases steadily with pressure. Such changes in the transition character under pressure are not uncommon; a well known example is the II-III transition in $\mathrm{NH}_{4} \mathrm{Cl}$ which goes the opposite way from being first order at atmospheric pressue to becoming second order above $0.2 \mathrm{GPa}$ [14]. The transformation between the tetragonal $14 \mathrm{~mm}$ phase and the high-pressure orthorhombic

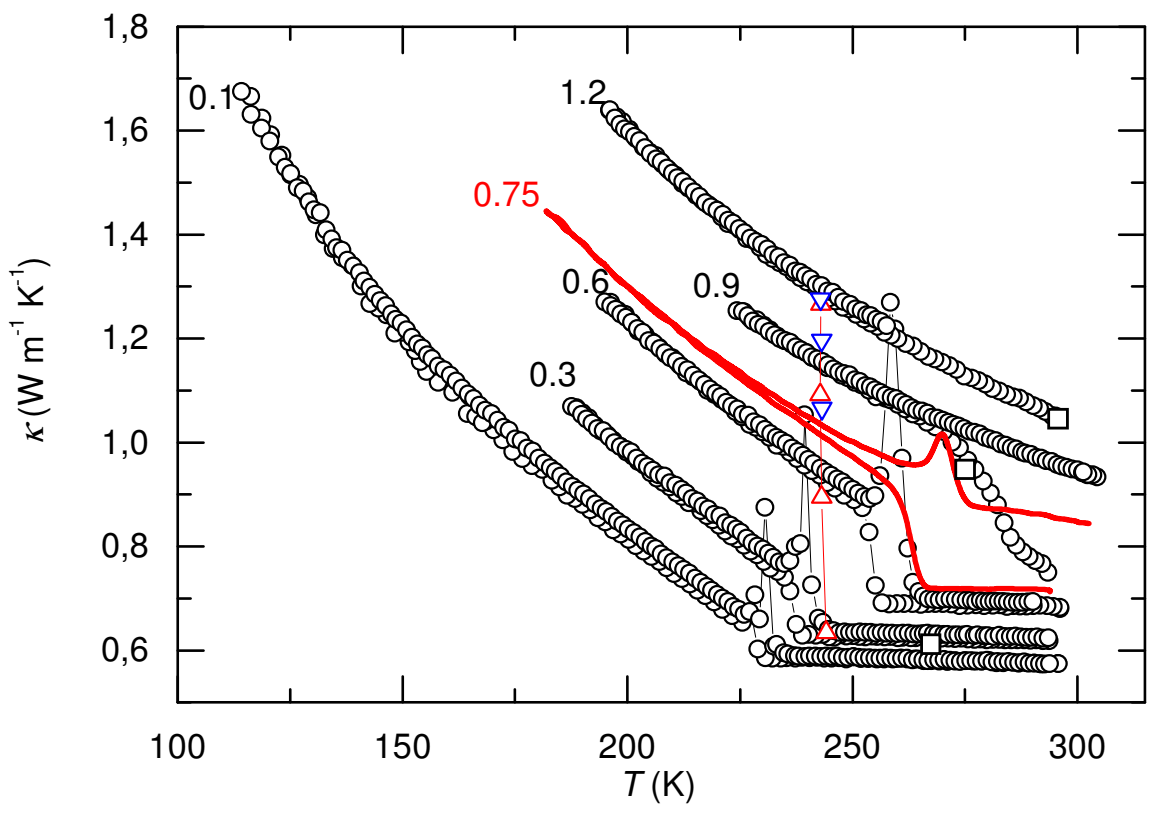

Figure 1. Thermal conductivity $\kappa$ as a function of temperature $T$ at the pressures (in GPa) indicated. 
$C m c 2_{1}$ phase was found to have a completely different character, with a very strong hysteresis. Again, there is a step increase in $\kappa$ on entering the ordered orthorhombic phase, whether by increasing pressure through the phase line at constant temperature or by cooling under isobaric conditions above $0.8 \mathrm{GPa}$. Once formed the $C m c 2_{1}$ phase was very stable, and at temperatures below $230 \mathrm{~K}$ some fraction could even be retained at atmospheric pressure. At $0.2 \mathrm{GPa}$ or higher it seemed to be completely stable over the time of the experiment at all temperatures in the stability region of the low temperature $P m n 2_{1}$ phase, as well as at all temperatures from 100 to $300 \mathrm{~K}$ above $1 \mathrm{GPa}$. In our experiments the reverse transformation back to the initial $14 \mathrm{~mm}$ phase did not occur until slightly below $0.5 \mathrm{GPa}$ at $293 \mathrm{~K}$, compared to 0.7-1 GPa in the experiments of Filinchuk et al. [8], and we could experimentally establish a reverse transformation phase line extending from this point down to just above $230 \mathrm{~K}$ at atmospheric pressure. At, or close to, atmospheric pressure, however, a partial transformation from the $C m c 2_{1}$ phase into the lowtemperature orthorhombic $P m n 2_{1}$ phase occurred, resulting in the observation of a double transformation at atmospheric pressure. The resulting phase diagram of $\mathrm{NH}_{3} \mathrm{BH}_{3}$ is shown in Figure 2.

In an intermediate region between approximately 0.65 and $0.8 \mathrm{GPa}$, isothermal cycling through the phase boundary resulted in the appearance of a high temperature phase with values of $\kappa$ falling between those of the tetragonal and orthorhombic phases of $\mathrm{NH}_{3} \mathrm{BH}_{3}$ (see Figure 1). Initially, we believed that these values might signify the discovery of the fourth high pressure,

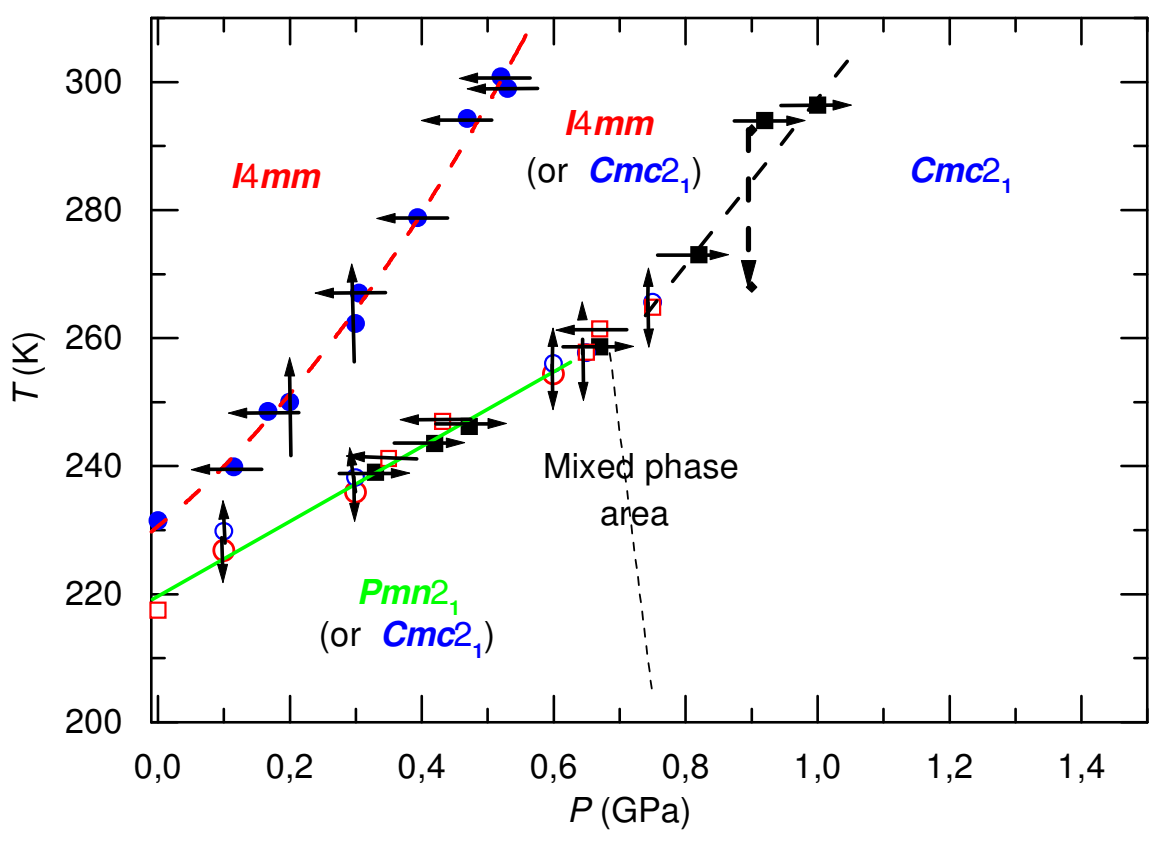

Figure 2. Pressure - temperature $(\mathrm{P}-\mathrm{T})$ phase diagram of $\mathrm{NH}_{3} \mathrm{BH}_{3}$ derived in the present work. Symbols show coordinates of the transformations observed, and arrows show both the transformation direction and the approximate transition ranges observed. Full green line denotes the $I 4 m m \leftrightarrow P m n 2_{1}$ transition and dashed lines transformations between $I 4 m m$ and $C m c 2_{1}$ phases. Dotted line indicates the approximate phase line for transformations from $P m n 2_{1}$ to $C m c 2_{1}$. 


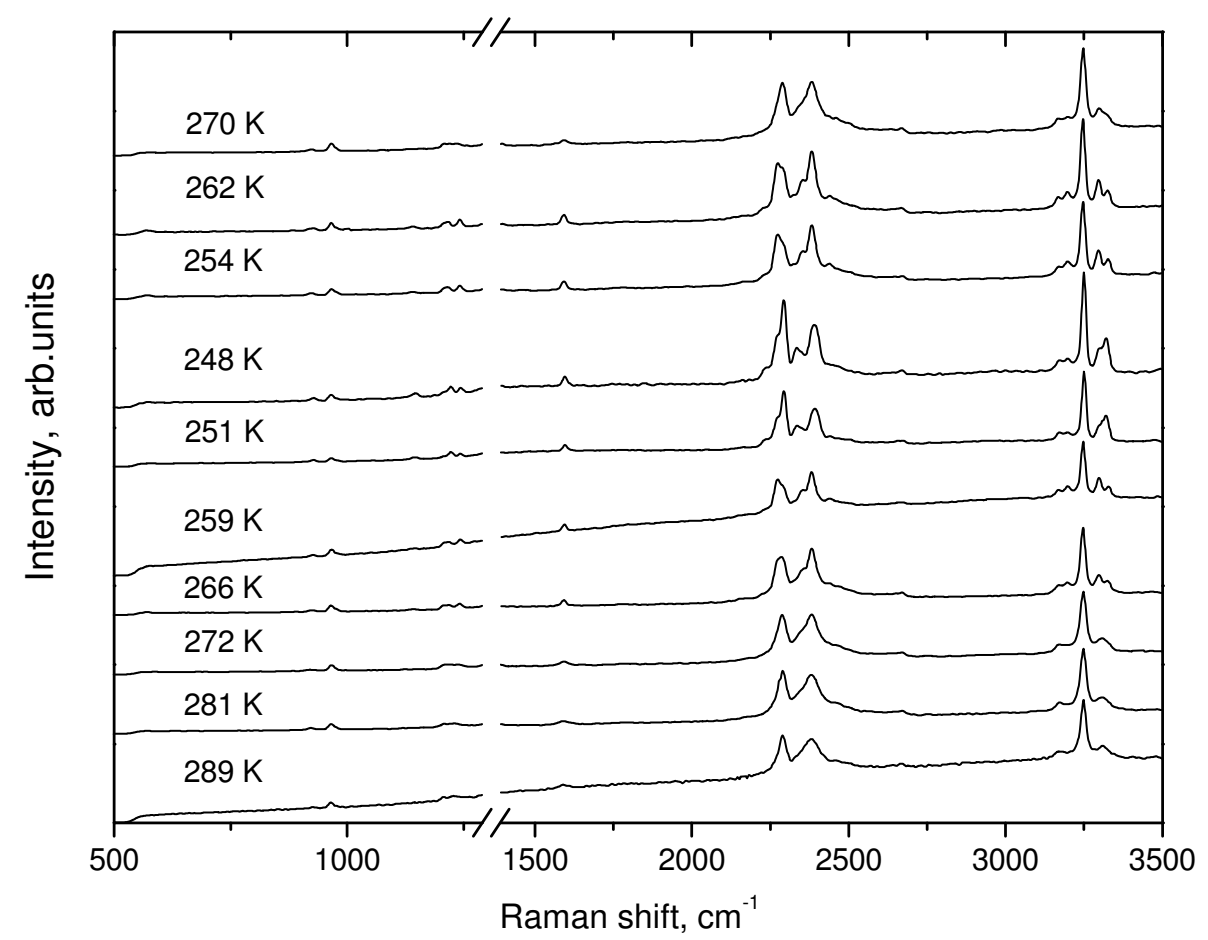

Figure 3. Raman spectra obtained in situ at $0.9 \mathrm{GPa}$ at the temperatures indicated. Spectra taken above and below $250 \mathrm{~K}$ show reversible differences indicating a phase transformation.

low temperature phase predicted by Filinchuk et al. [8]. However, Raman studies (Figure 3) at temperatures below the phase transition boundary in this pressure range suggested that cooling resulted in the formation of a mixture of the two orthorhombic phases. Heating back through the phase line then resulted in a transformation of the $P m n 2_{1}$ material into the low- $\kappa I 4 m m$ phase, while the metastable $C m c 2_{1}$ phase retained its low temperature structure, such that the resulting composite material displayed an intermediate value for $\kappa$.

\section{CONCLUSIONS}

We have been able to map the temperature-pressure phase diagram of $\mathrm{NH}_{3} \mathrm{BH}_{3}$ in detail in the range 100-300 K and 0-1.5 GPa. The phase transformation between the tetragonal $I 4 \mathrm{~mm}$ phase and the low temperature $P m n 2_{1}$ phase occurs at $218 \mathrm{~K}$ at atmospheric pressure and the phase boundary has a slope near $60 \mathrm{~K} \mathrm{GPa}^{-1}$. The transformation is associated with a small hysteresis in temperature or pressure, and the step change in $\kappa$ at the transition is very small at low pressures but increases continuously with increasing pressure. We interprete this as an evolution from a nearly second order transition at zero pressure toward first order at higher pressures. The phase boundary is approximately linear from atmospheric pressure up to about 0.6 
$\mathrm{GPa}$, where the transformation changes character and the phase transition boundary increases its slope significantly.

At room temperature we find the transition into the high-pressure $C m c 2_{1}$ phase near 1.1 $\mathrm{GPa}$, and the phase boundary delineated by the observed $I 4 m m \rightarrow C m c 2_{1}$ transformations connects this point and the high-pressure end of the observed $I 4 m m-P m n 2_{1}$ phase line. However, on decreasing pressure the $C m c 2_{1}$ phase is strongly metastable and the reverse transformation into tetragonal $14 \mathrm{~mm}$ occurs at significantly lower pressures (or higher temperatures). The reverse transformation points define a curve with an average slope near $140 \mathrm{~K} \mathrm{GPa}^{-1}$, extrapolating to $230 \mathrm{~K}$ at zero pressure. At and above $0.2 \mathrm{GPa}$ the $C m c 2_{1}$ phase seems to be stable at all temperatures below this line, but near atmospheric pressure a partial transformation into $P m n 2_{1}$ is observed below $220 \mathrm{~K}$. The strong metastability of the $C m c 2_{1}$ phase suggests a possibility to stabilize denser lattice structures, with higher volumetric hydrogen densities, in the $\mathrm{NH}_{3} \mathrm{BH}_{3}$ system by the addition of impurities or by other means.

\section{ACKNOWLEDGMENT}

Forskning.

This work was financially supported by Carl Tryggers Stiftelse för Vetenskaplig

\section{REFERENCES}

At the date this paper was written, URLs or links referenced herein were deemed to be useful supplementary material to this paper. Neither the authors nor the Materials Research Society warrants or assumes liability for the content or availability of URLs referenced in this paper.

1. L. Schlapbach and A. Züttel, Nature 414, 353 (2001).

2. Y. Filinchuk, D. Chernyshov, and V. Dmitriev, Z. Kristallogr. 223, 649 (2008).

3. B. Sundqvist, Solid State Phenom. 150, 175 (2009), http://www.scientific.net/SSP.150.175.

4. B. Sundqvist and O. Andersson, Int. J. Thermophys. 30, 1118 (2009).

5. L. George and S.K. Saxena, Int. J. Hydrogen Energy 35, 5454 (2010).

6. F.H. Stephens, V. Pons, and R.T. Baker, Dalton Trans. issue 25, 2613 (2007).

7. Y. Lin, W.L. Mao, V. Drozd, J.H. Chen, and L.L. Daemen, J. Chem. Phys. 129, 234509 (2008).

8. Y. Filinchuk, A.H. Nevidomskyy, D. Chernyshov, and V. Dmitriev, Phys. Rev. B. 79, 214111 (2009).

9. R.S. Kumar, X.Z. Ke, J.Z. Zhang, Z.J. Lin, S.C. Vogel, M. Hartl, S. Sinogeikin, L. Daemen, A.L. Cornelius, C.F. Chen, and Y.S. Zhao, Chem. Phys. Lett. 495, 203 (2010).

10. J. Nylén, T. Sato, E. Soignard, J.L. Yarger, E. Stoyanov, and U. Häussermann, J. Chem. Phys. 131, 104506 (2009).

11. B. Håkansson, P. Andersson, and G. Bäckström, Rev. Sci. Instrum. 59, 2269 (1988).

12. O. Andersson, A. Soldatov, and B. Sundqvist, Phys. Rev. B 54, 3093 (1996).

13. O. Andersson and H. Suga, Phys. Rev. B 65, 140201 (2002).

14. R.G. Ross and O. Sandberg, J. Phys. C: Solid State Phys. 12, 3649 (1979). 